\title{
A new approach of the constrained groove pressing process on Al5083-O alloy using PMMA polymer, without die non-friction coefficient: nanostructure, mechanical Properties and hardness
}

DOI:10.36909/jer.12957

\author{
Hadi. Tagimalek ${ }^{*}$, Mohammad Reza Maraki ${ }^{* *}$, Masoud Mahmoodi * \\ * Faculty of Mechanical Engineering, Semnan University, Semnan, Iran \\ ** Department of Materials and Metallurgy Engineering, Birjand University of Technology, Birjand, Iran \\ Corresponding author's E-mail: $\underline{\text { h_tagimalek@semnan.ac.ir, Maraki@ birjand.ac.ir }}$
}

\begin{abstract}
The Constrained Groove Pressing (CGP) on annealed Al5083 (Al5083-O) alloy sheets by means of Polymethyl Methacrylate (PMMA) polymer is investigated in this research. Input parameters include Traverse Speed (1, 2, and $3 \mathrm{~mm} / \mathrm{min}$ ), Pass Number (1, 2, 3, and 4), and Lubricants (Grease, Oil, and without lubricants). Reciprocally, output parameters consist of Nanostructure (SEM and EBSD images), Hardness (HV), and Mechanical Properties - Impact Strength $\left(\mathrm{kJ} / \mathrm{m}^{2}\right)$, Tensile Strength (MPa), Young's Modulus (MPa), and Elongation (\%). Revealed by the results, the impact strength is gradually improved in the $1^{s t}, 2^{\text {nd }}, 3^{r d}$, and $4^{\text {th }}$ passes by respectively adding oil and grease, compared to the state of using no lubricant. The impact strength results demonstrated that the addition of grease has a significant effect on the impact strength of composite samples. In order to build a high-quality product through the $C G P$ process, it is very important for the mechanical properties to be based on the input parameters. In CGP processes, the Continuous Dynamic Recrystallization $(C D R X)$ is considered as the ultrafine-grained mechanism. To predict the grain size evolution and the plastic deformation rate, the combination of Finite Element (FE) method and the ETMB model is utilized. Indicating the high generalizability and reliability as compared to other modeling methods, both the experimental test results and the ETMB model data, which have a higher degree of accuracy, are presented.
\end{abstract}

Keywords: Constrained groove process; Mechanical properties; Hardness; Impact strength; ETMB model; A15083-O; PMMA.

\section{INTRODUCTION}

In metallic materials, the grain size has a great influence on materials' strength and shaping, so it is necessary to study the microstructural alterations of metallic materials such as grain size variations and also investigate the relation between grain size and mechanical properties of metallic materials (André et al, 2016; and Farmanbar et al, 2019). There are several methods to improve materials' mechanical properties, such as the increment of strength and formability (Grujicic et al, 2008). In most of these methods, one mechanical property is improved, while the others are weak (Amancio-Filho et al, 2011 and Bozkurt, 2012). For example, the heat treatment is one of the ways to increase the steel's strength; as the steel is cooled down, the strength rises up, while the deformation capability is significantly reduced. So, fine-tuning the grain size of metallic materials 
is the only way to simultaneously improve both of the strength and deformation ability (Yangyang et al, 2021). The Severe Plastic Deformation (SPD) (Siahsarani et al, 2021), mechanical alloying (Sun et al, 2021), and thermomechanical processes (Yan et al, 2021) are some of the ways to reduce the grain size of polycrystalline materials. The SPD process is widely used to fine the grain sizes and improve the mechanical properties since they are simple and inexpensive (Alyani et al, 2021). Different types of SPD - namely Equal Channel Angular Pressing (ECAP) (Sadasivan et al, 2020), Accumulative Roll Bonding (ARB) (Khdair et al, 2020), Equal Channel Angular Rolling (ECAR) (Chen et al, 2020), and Constrained Groove Pressing (CGP) (Kumar et al, 2021) - have been proposed so far.

Nowadays, global competitive conditions have brought the obligation to produce high-quality products in the least time and expense. To meet this end, it is necessary to develop the integration of studies on optimization and prediction areas (Maraki et al and Mahmoodi et al; 2020), and also accelerate the development of new technologies relating to the design and manufacturing process. Increasingly growing the importance of these studies, new methods are being developed. Unlike to the laboratorial calculating methods such as the Finite Element $(F E)$ method (Tagimalek et al, 2020) and Image Processing (Tagimalek et al, 2021), that are much more affordable and timeefficient, the modeling, optimizing, predicting, and controling the complex systems, especially impressed by more than one parameter, are being employed in industrial applications (Pasoudeh et $a l, 2020)$. There is no laboratory error in the numerical simulation and evaluation processes, so they are excellent methods in investigating the microstructural alterations and mechanical properties of metallic materials. To predict the microstructure of materials during metal forming processes, several models such as the Cellular Automata (CA) (Majta et al, 2016), Monte Carlo (MC) (Li et al, 2010), Phase Field (Ishii et al, 2020), Molecular Dynamic Simulation (Teboul et al, 2005), and ETMB (Hosseini et al, 2009) are widely used. The parameters of the ETMB model are physical type parameters which related to the material.

The $C G P$ process of $A l 2024$ - with a high strength-to-weight ratio, at $300^{\circ} \mathrm{C}$, and within three cycles - was investigated by Ghorbanhosseini et al, in 2020. Calculating the Lankford coefficient, a normal and planar anisotropy was accomplished for samples which produced via $C G P$ process up to 3 passes. Based on texture analysis, it was found that the one-pass $C G P$ process can result in the most normal anisotropy which means the lowest fracture risk in the subsequent metal forming process such as deep drawing. The sheet's behavior was experimentally and numerically studied and examined by Nazari et al, in 2018, and also the CGP process was classified into three steps of bendings, stretching, and forging. Using plasticity connection, the process force was then estimated and the results were compared with the experimental data and $F E$ simulation. To investigate the effect of both material and geometry parameters, three different materials and two different geometries of die and sheet were compared together. According to the obtained results, presented correlations can predict the force during the CGP process for each type of material and geometry of sheet and die. Applying a combined isotropic-kinematic hardening model in the simulation, Kumar et al, in 2019, numerically surveyed the considered Bauschinger effect. The isotropic hardening, which is validated with the available results in the literature, is assumed by the $F E$ model. The $C G P$ experiments were established using three different materials and modeled using both isotropic and combined hardening models. The maximum strain and the strain inhomogeneity predicted by the combined hardening models were consistently greater than the isotropic hardening assumption. A paper titled "Using CGP Process Technique" was published by Khodabakhshi et al, in 2011, that is utilized for imposing SPD on low carbon steel sheets. The results proved that the CGP process can effectively refine a coarse-grained structure to an ultrafine-grained range. Dislocations' densities of ultrafine-grained low-carbon steel sheets are quantitatively calculated and it is found that the CGP 
process can effectively enhance the dislocations' densities of the sheets. The measurement of their electrical resistivity values indicates that the microstructure refinement and thr density increment of dislocations can efficiently increase the electrical resistivity of $C G P$ ed sheets up to about $100 \%$. As well, another paper titled "Using SPD Method Called CGP Process", which is used for imposing a high magnitude of strain into low carbon steel sheets, has been worked out by Khodabakhshi et al, in 2010. Employing X-Ray diffraction and optical observations, microstructural alterations during the process were examined. During the $S P D$ process, the grain size evolution was carried out using Williamson-Hall analysis on the X-Ray pattern of the deformed samples. The Ferrite grains with a submicron size of $200 \sim 300 \mathrm{~nm}$ as well as an ineffective strain of 4.64 were finally achieved. Shown by the result, the $C G P$ process can effectively refine a coarse-grained structure to an ultrafinegrained range. The mechanical properties were modified due to the measurement of microstructural evolution by both the tensile and hardness tests. The result expressed that the CGP process leads to a decrease in work hardening rate and an increase in strain rate sensitivity of the sheets' material. Ultimately, a deformed sheet with the grain size of $230 \mathrm{~nm}$ and the utmost tensile strength of 400 $M P a$ was obtained in a cumulative strain of 4.64 .

Checking the $F E+E T M B$ method, the entire mechanical properties, hardness and microstructural alterations - including the grain size and dislocations' density - are scrutinized in the current study through a new approach of the CGP process on $A 15083-O$ alloy using $P M M A$ polymer, without die friction coefficient.

\section{MATERIALS AND METHODS}

In the $C G P$ process, the structure is a momentous factor and plays the most crucial role in mechanical properties. Generating the local heat is one of the CGP functions. In fact, at the onset of die contact with the material, the heat generation is the first function due to the friction. All the parameters of angle, lubricant, traverse speed, and pass number are also important in terms of the heat generation. However, other design parameters have little effect on the generated heat. Given the important effect of die's traverse speed on the metal flow, the consequent microstructure, which is directly related to the grain size, will be varied by the changing in traverse speed, pass number, and lubricant.

The $C G P$ trajectory is perpendicular to the die length. It was accomplished in both sinuate and straightforward stages. At the first pass, an strain of about 0.58 was exerted on the sheets' deformed regions. A $C G P$ process with eight sinuate and straightforward die traverse is called a four-pass process. At last, a homogeneous effective strain of about 1.16 was produced throughout the sample.

The CGP die process is illustrated in Fig.1. After each two passes, the process includes a rotation of $90^{\circ}$, so it is able to diminish the anisotropic evolution problems of different properties during the process. 

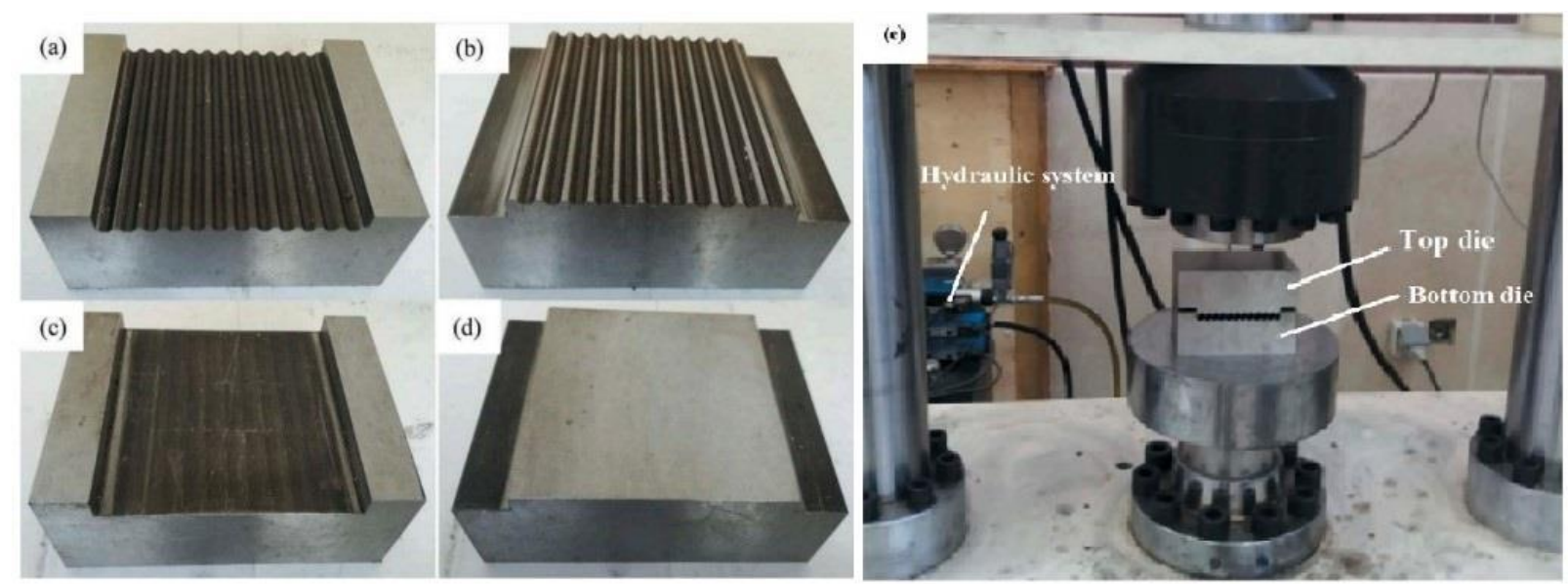

Fig.1: $C G P$ process on $A l 5083-O$ alloy using $P M M A$ polymer, without die non-friction coefficient

Table 1: Mechanical properties of pure PMMA and Al5083-O

\begin{tabular}{cccc}
\hline Sheet & Hardness (HV) & Impact strength $\left(\mathbf{k J} / \mathbf{m}^{\mathbf{2}}\right)$ & Young's modulus (MPa) \\
\hline Pure PMMA & 77.25 & 8.24 & 2899.3 \\
A15083-O & 137.5 & 22.7 & 76.5 \\
\hline
\end{tabular}

In the present research, some empirical studies on the CGP process of Al5083-O alloy have been conducted using PMMA polymer without die non-friction coefficient. One of the functions of three input parameters - traverse speed, pass number, and lubricant - was to change the mechanical properties. According to the ASTM E8-04 standard, the testing workpieces were prepared for the tensile test at a constant speed of $1 \mathrm{~mm} / \mathrm{min}$, an strain rate of 0.003 , and at the ambient temperature for all welded samples by the SANTAM STM-150. The sample's hardness was attained according to the ASTM-E384 standard and Vickers hardness test by the Bohler machine at a force of $250 \mathrm{gr}$ and a period of $10 \mathrm{~s}$. The Al5083-O samples were experimentally simulated via FEM. Besides using no lubricant, both grease and oil were used as lubricants between the pure PMMA and Al5083-O. For an $S E M$ microscopic observation, the samples were provided using an standard metallographic procedure, and then, the crosssection thickness was studied. To provide an SEM sample, a piece of sample was primarily plucked in the central part of the $C G P$ process's $1^{\text {st }}, 2^{\text {nd }}, 3^{\text {rd }}$, and $4^{\text {th }}$ passes. The mechanical properties of pure PMMA and Al5083-O are exhibited in Table 1.

\section{FINITE EIEMENT ANALYSIS}

The Abaqus software was utilized to simulate the CGP process. The upper and lower dies were considered to be analytically rigid while Al5083-O and pure PMMA sheets were considered to be elastic plastic. The sheet was meshed by some $S 4 R$ elements with the sizes of $1 \mathrm{~mm}$. The Columbus friction model and typical lubricants were grease $\sim 0.14$, oil $\sim 0.11$, and non-lubricant. Therefore, a dynamic explicit solvent was used owing to a large deformation in the cold state process.

To obtain the optimal size of mesh, the impact of element size on variations in Equivalent Plastic Strain (PEEQ) was investigated. As observed in Fig.2, by meeting the mesh size of 1.2, the smaller the size of mesh, the less impact on the PEEQ and Plastic Strain (PE). So, the mesh size of 1.2 was brought up for the sheet simulation. The impact of mesh size alteration on various strain changes of the centeral sample is depicted in Fig.2. 
One sample of $A l 5083-O$, with the thickness of $0.5 \mathrm{~mm}$, and two samples of pure PMMA, with the thickness of $2.5 \mathrm{~mm}$, were imposed to the $C G P$ process in the Abaqus software by $F E$ method. To sample the mesh, some $S 4 R$ elements with 20,000 were employed.

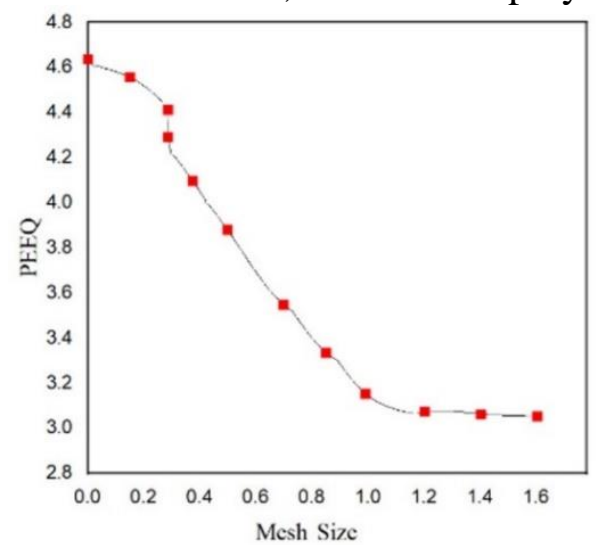

Fig. 2: Mesh Sensitivity Diagram: Effect of mesh size on PEEQ of composite sample

\section{ETMB model}

There are two categories in the matter of ETMB model. First, the element's dislocation; and second, the grain boundary. According to the rule of mixtures, dislocations' density of walls $\left(P_{w}\right)$, dislocations' density of elements $\left(P_{i}\right)$, and total dislocations' density $\left(P_{t}\right)$ are all presented in Eq.l.

$$
\rho_{\mathrm{t}}=\mathrm{f}_{\text {total }} \rho_{\mathrm{w}}+\left(1-\mathrm{F}_{\text {total }}\right) \rho_{\mathrm{i}}
$$

$F_{\text {total }}$ stands for the walls' volume percentage. This factor hits the peak by deformation changes, which can be expressed in Eq.2.

$$
\mathrm{F}_{\text {total }}=\mathrm{F}_{\infty}+\left(\mathrm{F}_{0}-\mathrm{F}_{\infty}\right) \exp \left(-\eta_{1} \gamma_{\mathrm{r}}\right)
$$

$F_{0}$ and $F_{\infty}$ values are related to the beginning of the process, contrary to $F_{\text {total }}$ which is the culmination value. As well, $\eta i$ is assumed as a numerical constant. Regarding to three mechanisms of dislocations' density change - namely the increment of dislocations by Frank-Reed sources, the dislocations' departure to other areas, and dislocations caused by discharge recovery process - the dislocations' density of cells and walls are represented in Eqs. 3,4.

$$
\begin{gathered}
\frac{\mathrm{d} \rho_{\mathrm{i}}}{\mathrm{dt}}=\frac{\alpha^{*} \dot{\gamma}_{\mathrm{w}} \sqrt{\rho_{\mathrm{w}}}}{\sqrt{3} \mathrm{~b}}-\frac{6 \beta^{*} \dot{\gamma}_{\mathrm{i}}}{\mathrm{bd}(1-\mathrm{F})^{\frac{1}{3}}}-\mathrm{R}_{\mathrm{i}} \dot{\gamma}_{\mathrm{i}} \rho_{\mathrm{i}}\left(\frac{\dot{\gamma_{\mathrm{i}}}}{\dot{\gamma}_{0}}\right)^{\frac{-1}{\mathrm{n}_{\mathrm{i}}}} \\
\frac{\mathrm{d} \rho_{\mathrm{w}}}{\mathrm{dt}}=\frac{6 \beta^{*} \dot{\gamma}_{\mathrm{i}}\left(1-\mathrm{F}_{\text {total }}\right)^{\frac{2}{3}}}{\mathrm{bd} \mathrm{F}_{\text {total }}}+\frac{\sqrt{3} \beta^{*} \dot{\gamma}_{\mathrm{i}}\left(1-\mathrm{F}_{\text {total }}\right) \sqrt{\rho_{\mathrm{w}}}}{\mathrm{F}_{\text {total }}}-\mathrm{R}_{\mathrm{w}} \dot{\gamma}_{\mathrm{w}} \rho_{\mathrm{w}}\left(\frac{\dot{\gamma_{\mathrm{w}}}}{\dot{\gamma}_{0}}\right)^{\frac{-1}{\mathrm{n}_{\mathrm{w}}}}
\end{gathered}
$$

In this equation, $\alpha^{*}$ and $\beta^{*}$ are as for dislocations' production parameters; $\dot{\gamma}_{i}$ and $\dot{\gamma}_{w}$ are concerned with equivalent shear strain rates; Burgers' vector is shown by letter $b ; R_{i}$ and $R_{w}$, are put as coefficients of dynamic recovery process; and finally, $\dot{\gamma}_{r}, \eta_{i}$, and $\eta_{w}$ as the model's constants. The ratio of average grain size to total dislocations' density is provided in Eq.5.

$$
\mathrm{d}=\frac{\mathrm{K}}{\sqrt{\rho_{\mathrm{t}}}}
$$


Equations (5) and (6) are two first-degree differential equations, which solved numerically by the values of dislocations' density of cells and walls as well as the total dislocations' density. The symbol of $K$ in Eq.6 is the grain size coefficient, which decreases once the accumulation of deformation comes to the climax.

$$
\mathrm{K}=\mathrm{K}_{\infty}+\left(\mathrm{K}_{0}-\mathrm{K}_{\infty}\right) \exp \left(-\eta_{2} \gamma_{\mathrm{r}}\right)
$$

In Eq.7, the symbols of $K_{0}, K_{\infty}$ and $f_{\infty}$ are respectively the $K$ value at the beginning of the process and also the $K$ value of saturation. The symbol of $\eta_{2}$ is also a numerical constant. The material's flow rate is derived from the following.

$$
\sigma_{\mathrm{y}}=\sigma_{0}+\alpha \mathrm{MGb} \sqrt{\rho_{\mathrm{t}}}
$$

$\sigma_{y}, \sigma_{0}, M$ and $G$ in Equation (7) respectively stand for the flow stress, yield stress, Taylor's factor and shear modulus of the material. $\alpha$ is a constant of matter too. All model parameters and constants related to the SPD process and PMMA and Al5083-O sheets have been extracted from the previous research. The $C G P$ process simulation of one, two, three and four-pass samples, by integrating the ETMB model, is demonstrated in Fig.3.

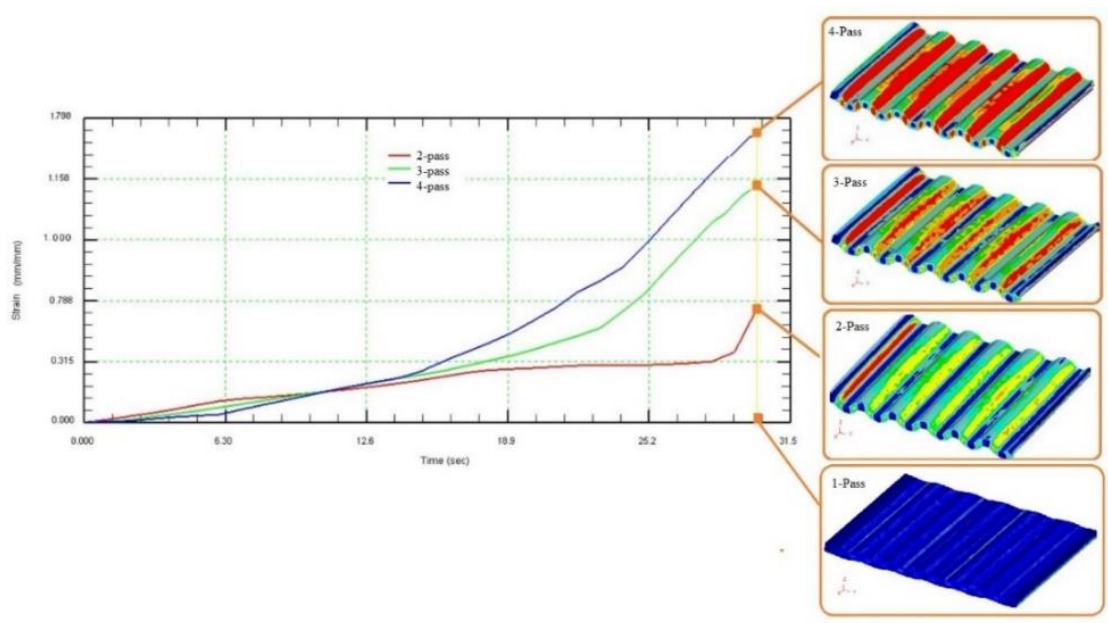

Fig. 3: $C G P$ process simulation of one, two, three and four-pass sample sheets, by integrating ETMB model

\section{RESULTS AND DISCUSSION}

\section{Mechanical properties and hardness}

In the present study, the mechanical properties - i.e. impact strength, tensile strength, Young's modulus, elongation, and hardness - of the Al5083-O samples have been considered. Due to the friction between the Al5083-O sample and non-friction die using PMMA polymer, the strain at the sample's surface had been theoretically expected to be lower than its center, which was in a good agreement with Fig.3. So, the effective strain at the center of sample was higher than its surface.

For the effect of both friction and hardness, the strain distribution was very heterogeneous. The mechanical properties of Al5083-O samples including the Young's modulus, impact strength, and hardness were analyzed. The results revealed that the impact strength is improved in the $1^{\text {st }}, 2^{\text {nd }}$, $3^{\text {rd }}$, and $4^{\text {th }}$ passes by respectively adding oil and grease, compared to the state of using no lubricant. 
It was proved that the addition of grease has a significant effect on the impact strength of Al5083-O samples. One, two, three, and four-pass $C G P$ processes in presence of grease and oil, which has a noteworthy effect on the impact strength, are indicated in Fig.4.

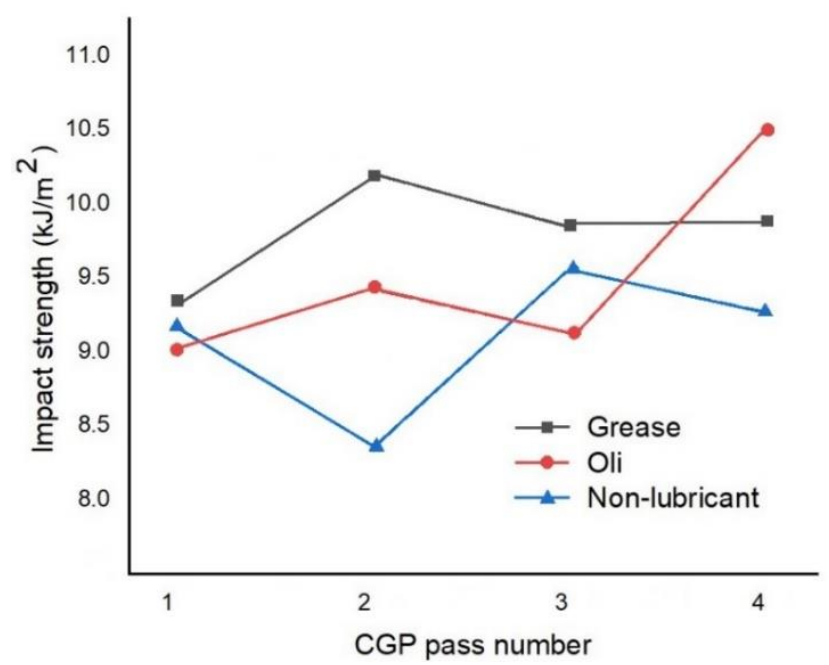

Fig 4: Effect of the $C G P$ pass number on impact strength in presence of grease, oil and without lubricants

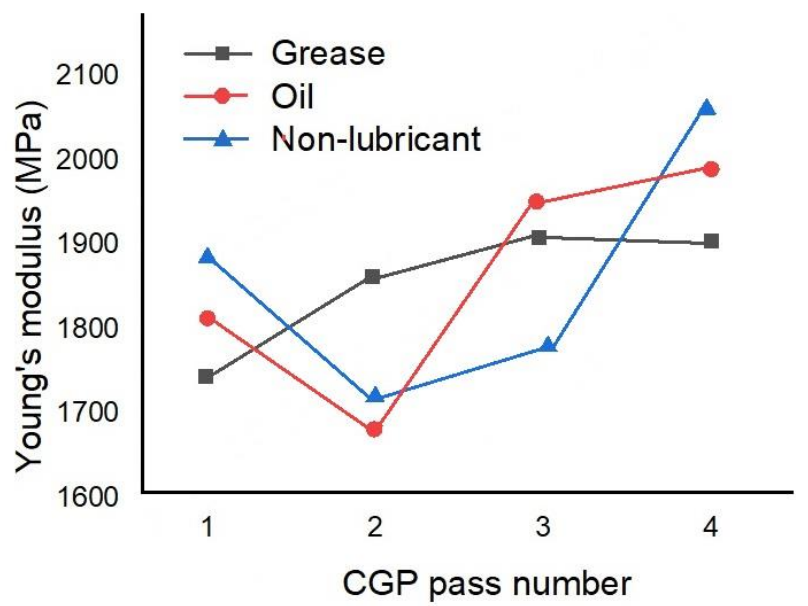

Fig 5: Effect of the CGP pass number on Young's modulus in presence of grease, oil and without lubricants 


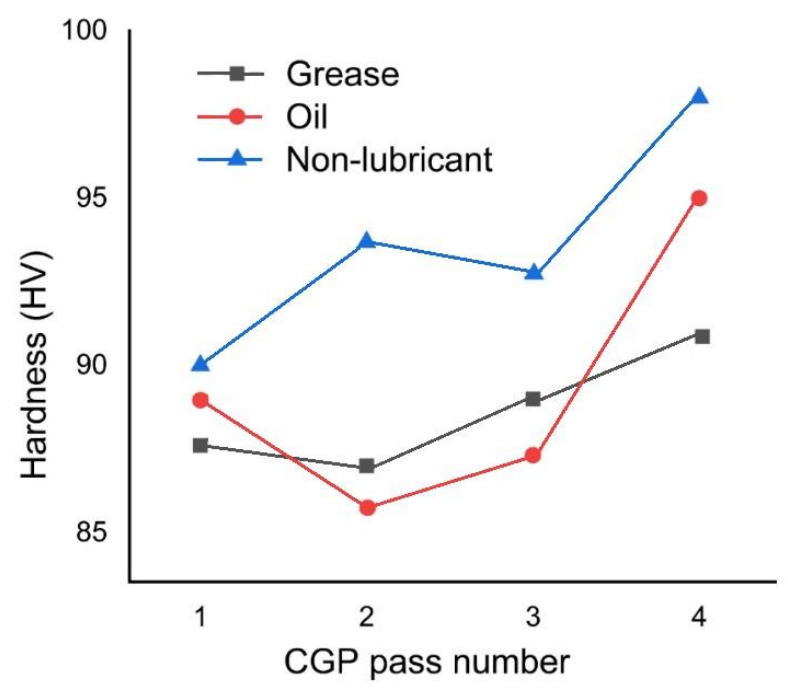

Fig 6: Effect of the CGP pass number on hardness in presence of grease, oil and without lubricants

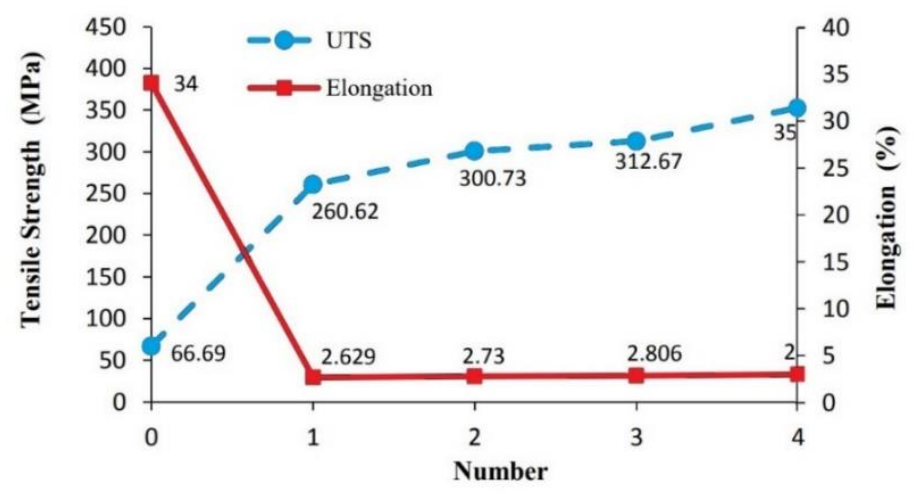

Fig 7: Effect of the $C G P$ pass number on tensile test

Due to the hard-work phenomenon, the sample's hardness increases as it moves within the die. Applyed strain to the sample, some misalignments are produced. So, applying strain leads to a more aberrant production, and thus, the rise of sample's strength. The tensile test results, impact strength, Young's modulus, and hardness of samples are displayed in Figs. 4,5,6,7. Grease has the most impact on the Young's modulus and tensile test of Al5083-O sheets during four passes. Another important result that can be obtained from Fig.5 is the fact that the young's modulus and the hardness of samples are improved by the CGP's pass number and lubricant type of the Al5083-O sheet.

\section{Micro and Nano-structure}

The analysis of die non-friction coefficient in the Al5083-O alloy, using PMMA polymer, was evaluated by $S E M$ images. The obtained images and the grafting boundary of Al5083-O alloy are shown in Fig.8. According to the results, a suitable and acceptable grafting is achieved in the process. The SEM image of diffraction in the Al5083-O alloy sample is depicted in Fig.9. The grafting boundary indicates that the consequent grafting is approved. As observed in the solid-state, the grafting boundary of $A 15083-O$ and used PMMA was homogeneous. 

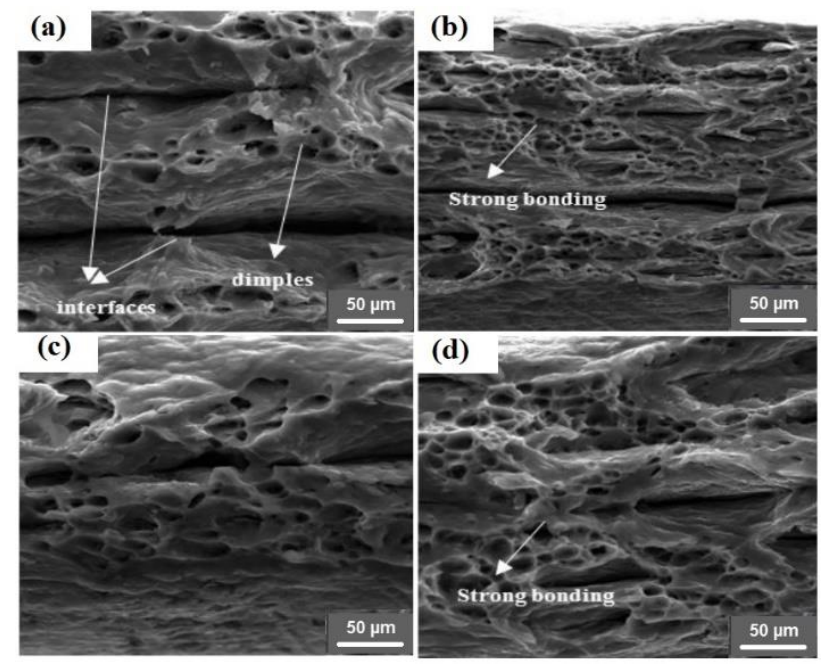

Fig 8: SEM cross-section image of Al5083-O using PMMA sheet: a) one-pass, b) two-pass, c) three-pass, and d) four-pass
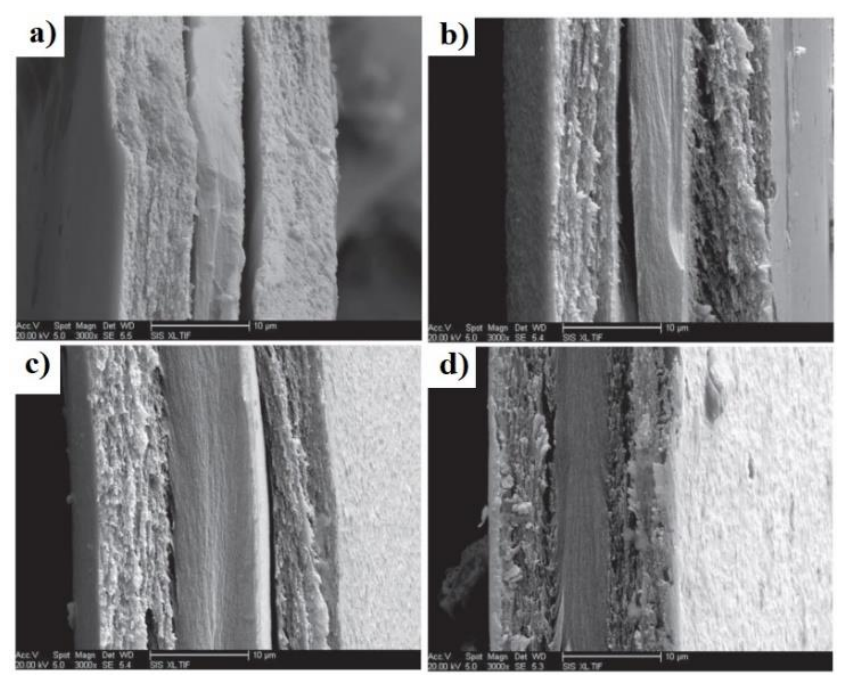

Fig 9: SEM images; microstructure of Al5083- $O$ using PMMA sheet: a) one-pass, b) two-pass, c) three-pass, and d) four-pass

To further analyze the grain morphology and crystallographic texture, the Inverse Pole Figure (IPF) Electronic Back-Scatter Diffraction (EBSD) maps of given CGPed samples are acquired, which presented in Fig10. The equiaxed grain structure can be observed in the layer between Al5083-O and PMMA, and this is while the grains at the inner layer region uncover a larger grain size. The characteristics of columnar structure are present at the inner layer region and equiaxed grains can be witnessed at the interlayer region of the Al5083-O samples.

There is a Continuous Dynamic Recrystallized (CDRX) grain size shrinkage in the SPD method, in which this mechanism was formed by applying strain to the material, and also small byproducts fabricated and eventually deformed. These are the main grains that cause the size of polycrystalline materials to be shrinked. The grain size variations during the CGP process of Al5083-O, without die non-friction coefficient, are demonstrated in Fig.11. The grain size decreases with increasing strain at the initial strain and after saturation, it becomes final, which means that strain size can be finely reduced by applying strain. 

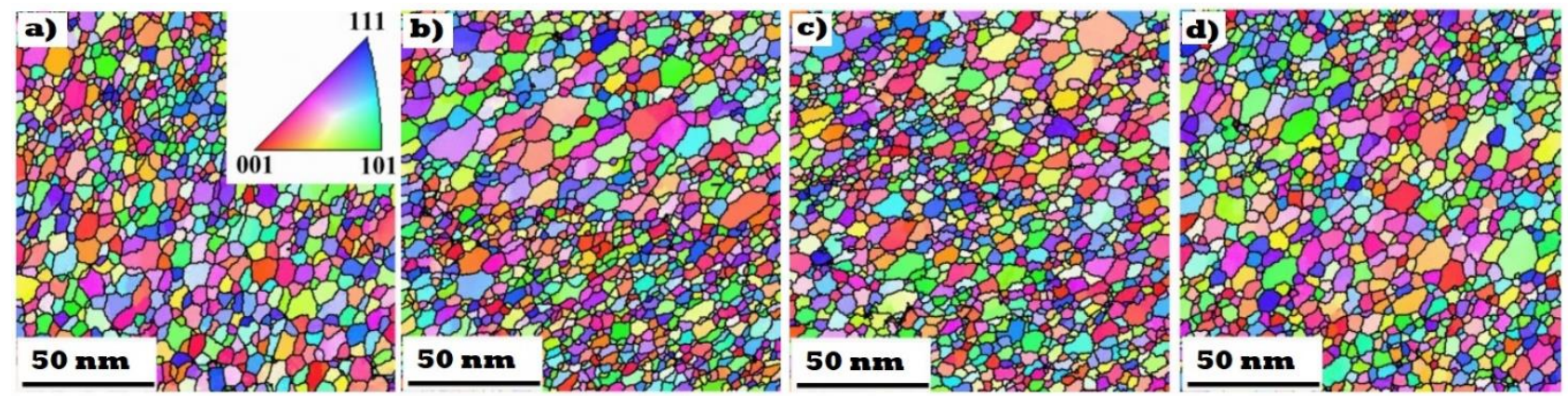

Fig 10: The centeral region of Al5083-O; IPF maps of these four samples: a) one-pass, b) two-pass, c) three-pass and d) four-pass

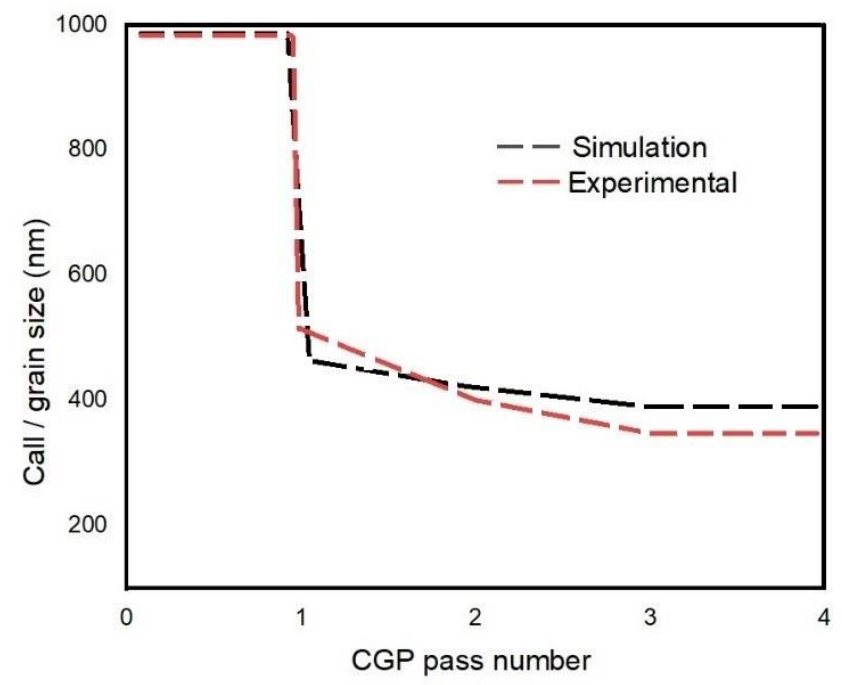

Fig 11: Grain size variations in the $C G P$ pass of $A l 5083-O$

\section{CONCLUSION}

A new approach concerning nanostructure, mechanical properties, and hardness in the Constrained Groove Pressing (CGP) process on Al5083-O alloy using PMMA polymer, without die non-friction coefficient, is investigated in this paper via the experimental results and simulation of $F E+E T M B$ models. Input parameters including the traverse speed, the pass number, and the lubricants are measured, and obtained results are as the following:

- The CGP process increases both the tensile strength and yield strength with the highest growth in the first pass and it continues to the extreme strength with a lower slope in subsequent passes.

- According to the Von-Mises Yield Criterion, instability and choking start earlier in constrained groove samples and this indicates a low hardness rate. As compared to the base sample, the rise of heterogeneous deformations in the constrained groove samples indicates an increase in sensitivity of the strain rate material as well.

- In reliability and generalization, the ETMB model has a higher level than the other methods of dislocation model. 
- In a satisfactory compliance with the experiment results, The strain and grain sizes are calculated and predicted by the ETMB model.

- In the CGP process, the strain is extremely homogeneous, indicating that the cutting process is simple.

- At the beginning of the process, the density of dislocations is almost constant; but, it further increases after the strain and eventually culminates at the end of the process.

- Increasingly processed at the first, the sample's grain size is going to reached the pinnacle over time.

\section{ACKNOWLEDGMENT}

The authors thank Ms. Parisa Nazari at the Memorial University of Newfoundland, for providing the authors group with free EBSD maps.

\section{REFERENCES}

André, N.M., Goushegir, S.M., Dos Santos, J.F., Canto, L.B., \& Amancio-Filho, S.T. 2016. Friction Spot Joining of aluminum alloy 2024-T3 and carbon-fiber-reinforced poly (phenylene sulfide) laminate with additional PPS film interlayer: Microstructure mechanical strength and failure mechanisms. Composites Part B: Engineering, 94: 197-208.

Farmanbar, N., Mousavizade, S.M., \& Ezatpour, H.R. 2019. Achieving special mechanical properties with considering dwell time of AA5052 sheets welded by a simple novel friction stir spot welding. Marine Structures. 65: 197-214.

Grujicic, M., Sellappan, V., Omar, M.A., Seyr, N., Obieglo, A., Erdmann, M., \& Holzleitner, J. 2008. An overview of the polymer-to-metal direct-adhesion hybrid technologies for load-bearing automotive components. Journal of Materials Processing Technology. 197(1-3): 363-373.

Bozkurt, Y. 2012. The optimization of friction stir welding process parameters to achieve maximum tensile strength in polyethylene sheets. Materials \& Design. 35: 440-445.

Amancio-Filho, S.T., Bueno, C., Dos Santos, J.F., Huber, N., \& Hage Jr, E. 2011. On the feasibility of friction spot joining in magnesium/fiber-reinforced polymer composite hybrid structures. Materials Science and Engineering: A. 528(10-11): 3841-3848.

Yangyang. L., Dong. Li., Heng. Ma., Xiliang, Liu., Meihong, Wu., \& Jing, Hu. 2021. Enhanced plasma nitriding efficiency and properties by severe plastic deformation pretreatment for $316 \mathrm{~L}$ austenitic stainless steel. Journal of Materials Research and Technology. 15: 1742-1746.

Siahsarani, A., \& Faraji, G. 2021. Processing and characterization of AZ91 magnesium alloys via a novel severe plastic deformation method: Hydrostatic cyclic extrusion compression (HCEC). Transactions of Nonferrous Metals Society of China. 31(5): 1303-1321.

Sun, C., Guo, Y., Yang, Z., Li, J., Xi, S., Jie, Z., \& Xu, T. 2021. Microstructurally stable nanocomposite $\mathrm{WTaMoNb} / \mathrm{Cu}$ prepared by mechanical alloying and hot pressing sintering. Materials Letters. 306: 130894.

Yan, B., Zhang, Y., Jiang, S., Yu, J., Sun, D., \& Tang, M. 2021. Mechanical properties and fracture mechanisms of martensitic NiTi shape memory alloy based on various thermomechanical-processing microstructures. Journal of Alloys and Compounds. 883: 160797.

Alyani, A., \& Kazeminezhad, M. 2021. Annealing behavior of aluminum after low-temperature severe plastic deformation. Materials Science and Engineering: A. 824: 141810.

Sadasivan, N., Balasubramanian, M., \& Rameshbapu, B.R. 2020. A comprehensive review on equal channel angular pressing of bulk metal and sheet metal process methodology and its varied applications. Journal of Manufacturing Processes. 59: 698-726. 
Khdair, A.I., \& Fathy, A. 2020. Enhanced strength and ductility of Al-SiC nanocomposites synthesized by accumulative roll bonding. Journal of Materials Research and Technology. 9(1): 478-489.

Chen, Y., Hu, L., Shi, L., Zhou, T., Tu, J., Chen, Q., \& Yang, M. 2020. Effect of texture types on microstructure evolution and mechanical properties of AZ31 magnesium alloy undergoing uniaxial tension deformation at room temperature. Materials Science and Engineering: A. 769: 138497.

Kumar, S., \& Vedrtnam, A. 2021. Experimental and numerical study on effect of constrained groove pressing on mechanical behaviour and morphology of aluminium and copper. Journal of Manufacturing Processes. 67: 478-486.

Maraki, M.R., Tagimalek, H., Azargoman, M., Khatami, H., \& Mahmoodi, M. 2020. Experimental Investigation and Statistical Modeling of the Effective Parameters in Charpy Impact Test on AZ31 Magnesium Alloy with V-shape Groove Using Taguchi Method. International Journal of Engineering, Transactions C: Aspects. 33(12): 2521-2529.

Mahmoodi, M., Tagimalek, H., Sohrabi, H, \& Maraki, M.R. 2020. Using the artificial neural network to investigate the effect of parameters in square cup deep drawing of aluminum-steel laminated sheets. International Journal of Iron \& Steel Society of Iran. 17(2): 1-13.

Pasoudeh, B., \& Tagimalek, H. 2020. Analytical and Numerical Evaluation of Wire Flat Rolling Process Based on the Slab Method and DEFORM-3D. Journal of Advanced Materials and Processing. 8(4): 316.

Tagimalek, H., Maraki, M.R. Mahmoodi, M., \& Mohammadzadeh, P. 2021. Investigation Experimental and Finite Element Method of Mechanical Properties of Hot Forging on Ti6Al4V Alloy. Iranian Journal of Energy and Environment (IJEE). 12(2): 149-154.

Tagimalek, H., Azargoman, M., Maraki, M.R., \& Mahmoodi, M. 2020. The effects of diffusion depth and heat-affected zone in NE-GMAW process on SUH 310S steel using an Image processing method. International Journal of Iron \& Steel Society of Iran. 17(1): 11-20.

Majta, J., Madej, L., Svyetlichnyy, D.S., Perzynski, K., Kwiecien, M., \& Muszka, K. 2016. Modeling of the inhomogeneity of grain refinement during combined metal forming process by finite element and cellular automata methods. Materials Science and Engineering: A. 671: 204-213.

Li, Y., Feng, X.Q., Cao, Y.P., \& Gao, H. 2010. A Monte Carlo form-finding method for large scale regular and irregular tensegrity structures. International Journal of Solids and Structures. 47(14-15): 18881898.

Ishii, A. 2020. Energetics of heterogeneous $\mathrm{Mg}\{1012\}$ deformation twinning migration using an atomistically informed phase-field model. Computational Materials Science. 183: 109907.

Teboul, V., \& Alba-simionesco, C. 2005. Dynamical heterogeneity in glass-forming toluene: Comparison of bulk and confined conditions by quasielastic neutron scattering and molecular dynamics simulations. Chemical Physics. 137(2-3): 245-252.

Hosseini, E., Kazeminezhad, M. 2009. ETMB model investigation of flow softening during severe plastic deformation. Computational Materials Science. 46(4): 902-905.

Ghorbanhosseini, S., Fereshteh-Saniee, F., \& Sonboli., A. 2020. An experimental investigation on the influence of elevated-temperature constrained groove pressing on the microstructure, mechanical properties, anisotropy and texture of $2024 \mathrm{Al}$ sheets. Journal of Alloys and Compounds. 817: 152763.

Nazari, F., \& Honarpisheh., M. 2018. Analytical model to estimate force of constrained groove pressing process. Journal of Manufacturing Processes. 32: 11-19.

Kumar, S., Hariharan, K., Digavalli, R.K., \& Paul, S.K. 2019. Accounting Bauschinger effect in the numerical simulation of constrained groove pressing process. Journal of Manufacturing Processes. 38: 49-62.

Khodabakhshi, F., \& Kazeminezhad, M. 2011. The effect of constrained groove pressing on grain size, dislocation density and electrical resistivity of low carbon steel. Materials \& Design. 32(6):3280-3286.

Khodabakhshi, F., Kazeminezhad, M., \& Kokabi, A.H. 2010. Constrained groove pressing of low carbon steel: Structure and mechanical properties. Materials Science and Engineering: A. 527(16-17), 40434049. 
